homologuer ces deux facteurs à des mutants déjà observés chez la Souris domestique. Le premier variant peut être assimilé à "chinchilla " $\left(c^{\mathrm{ch}}\right)$ au locus albinos. Le second peut être rapproché d'un allèle de la série "pink eyed " $(p)$. Pour tester l'hypothèse d'un linkage de ces deux facteurs, nous avons mis en œuvre une première expérience qui, limitée par le temps et par l'espace disponibles, a consisté en l'examen de sujets $\mathrm{F}_{2}$. Pour la détermination de la couleur du pelage, on a utilisé une échelle colorée, mais cette méthode ne s'étant pas révélée suffisante pour la totalité des animaux à yeux rouges, nous avons dû recourir à des test-cross. Dans ces test-cross, qui devaient permettre de distinguer les doubles homozygotes $p p c^{\mathrm{ch}} c^{\mathrm{ch}}$ des individus $p p c^{\mathrm{ch}+}$ ou $p p++$ à pelage clair, on accouplait les sujets litigieux à des homozygotes "chinchilla" $++c^{\mathrm{ch}}$ $c^{\mathrm{ch}}$. Le nombre minimum de descendants ayant été fixé à huit, on avait une chance sur 256 de se tromper sur le génotype de l'animal soumis au test. On a obtenu 41 portées $F_{2}$ totalisant 267 sujets : 144 animaux de phénotype sauvage, 55 à yeux noirs et pelage clair, 52 à yeux rouges et pelage sauvage et 16 double récessifs. La probabilité du $\chi^{2}$ testant l'hypothèse d'indépendance (rapport théorique des phénotypes : $9: 3: 3: 1$ ) est supérieure à 0,80 . Dans ces conditions, on peut admettre provisoirement que les loci marqués par ces deux mutants sont génétiquement indépendants.

\title{
ENQUÊTE SUR LE CARACTÈRE CULARD DANS LA ZONE CHAROLAISE
}

B. VISSAC.- Station de génétique quantitative et appliquée, C.N.R.Z., 78-Jouy-en-Josas. J.-J. LAUVERGNE. - Station centrale de Génétique animale, C.N.R.Z., 78-Jouy-en-Josas I.N.R.A.

Cette étude a été réalisée en 1962 et 1963 dans 17 élevages charolais du département de Saône-et-Loire où étaient apparus fréquemment des veaux culards au cours des années antérieures. Le caractère culard a été apprécié subjectivement en Avril, Juillet et Septembre pour la totalité des veaux nés dans ces élevages $(\mathrm{N}=513)$ suivant une table en 10 points précédemment décrite. Les animaux ont été répartis arbitrairement en 3 catégories d'après la valeur de la note obtenue (0-20) : 0-6 : veaux normaux; 7-12 : veaux "semi-culards "; 13-20: veaux culards. Le pourcentage de veaux pointés comme culards (toutes périodes) était de 11,8 p. 100 en 1962 , de 4,0 p. 100 en 1963; celui des veaux culards et semi-culards de 34,6 p. 100 et 29,4 p. 100 respectivement. L'influence de l'année $(P<0,01)$ sur la fréquence de ces 2 catégories est interprétée par une incidence de l'opérateur. On a par ailleurs montré que le pourcentage de veaux notés culards et semi-culards variait suivant le sexe $(P<0,01)$, l'âge des veaux $(P<0,01)$, la période de contrôle $(P<0,01)$ et le $n^{\circ}$ de vêlage des femelles $(P<0,01)$. Aucun de ces facteurs $n^{\prime}$ avait par contre une incidence statistiquement notable sur le pourcentage de veaux vraiment culards $(P>0,05)$. Leur action sur la croissance musculaire de veaux normaux qui ont pu être confondus avec des semi-culards explique en partie ce résultat. Les différences entre descendances de taureaux apparaissent tant en 1962 qu'en 1963 dans la fréquence des veaux culards $(P<0,01)$ ainsi que dans celle des veaux culards et semi-culards $(P<0,01)$. Du fait de la pratique générale de la monte naturelle dans ces élevages, ces différences ont pu être accentuées par les variations de milieu entre troupeaux. On note, de même, une différence significative entre femelles dans la prédisposition à fournir des veaux culards $(\mathrm{P}<0,01)$ et culards ou semi-culards $(\mathrm{P}<0,01)$. La fréquence d'apparition d'un veau culard dans la descendance d'une femelle une année est environ 2 fois plus élevée si cette femelle a fourni un veau culard l'année précédente, que la fréquence correspondant à une femelle ayant donné la même année un veau normal. On a précisé également l'attitude des éleveurs vis-à-vis de la sélection des femelles présentant le caractère culard : aucun veau culard né en 1962 et 1963 n'a été mis à la reproduction. En ce qui concerne les deux autres catégories, on a pu noter une utilisation préférentielle des femelles de phénotype intermédiaire.

Ce texle résume une partie du mémoire de fin d'études rédigé sous notre direction par $M$. de La Guérivière (Institut technique de Pratique agricole, 1964, ronéoté). 